\title{
One Tale of Why and How to Teach and Learn O nline Internationally
}

\section{by Yu-Chih Doris Shih and Lauren Cifuentes}

$\mathrm{T}$ wo university instructors on opposite sides of Earth felt the need to have their students use telecommunications to facilitate learning in their face-to-face classes. They joined forces to prepare U.S. pre-service teachers for teaching online and reaching diverse learners, and to provide English instruction to Taiwanese students. Those geographically separated university students corresponded via email and a Web board. U.S. pre-service teachers explored theory and practice of online instruction, corresponded as tutors to teach English language and A merican culture, and reflected upon their experiences. Taiwanese students practiced English and exchanged cultural information. Here, we provide a rationale for online instructional practice in teacher education and in foreign language learning and then summarize the online teaching and learning strategies applied by the partners in this telecommunications partnership.

\section{Why Is 0 nune Teaching and LEARN ING So IMPORTANT?}

A s telecommunications become avail- able in schools, teachers need to be able to use them to serve their students. Schools are rapidly acquiring necessary hardware and software so that teachers and their students can apply telecommunications to teaching and learning. In 1995, only three percent of school classrooms, labs and media centers had Internet connections. A 1999 study, however, indicated that $57 \%$ of U.S. classrooms had direct access to the Internet (Q uality Education Data, 1999).

Romiszowski and Ravitz (1997) investigated teachers' perceptions of electronic teaching and found that the reward is "tremendous satisfaction in working towards the goal of developing independent, questioning learners" (p. 673).

Communication between teachers and students can be enhanced through computer conferencing on W eb boards and through e-mail connections. For instance, teachers can answer students' questions and provide brief tutorials for students who are having difficulties.
In addition to learning how to teach online, teachers need to be prepared to address the needs of students from diverse cultures. A s school demographics change, teachers are confronted with the need to teach students of differing cultural influences and encourage academic achievement for all types of learners. A study of Texas employers identified a lack of skills associated with multicultural awareness in college graduates, including those from teacher education programs. Requisite skills cited in the study included written and

\section{In addition to learning how to teach online, teachers need to be prepared to address the needs of students from diverse cultures.}

verbal presentation skills for diverse audiences, acknowledgement of multiple perspectives, application of sound judgment, ability to work in multicultural teams, flexibility in adapting to change, and ethical decision making. Such skills are increasingly required for success in 
teaching. In preparation for teaching diverse students and for multicultural awareness, pre-service teachers (PSTs) can expand their experiences with multicultural learners by using the Internet to communicate and collaborate with students from diverse backgrounds.

Therefore, teacher preparation programs are well advised to include online, supervised teaching experiences using telecommunications. Typically, PSTs have never taught online and few have experienced formal online learning. They have no models or knowledge with which to link the new experience of online teaching. A constructivist view of learning suggests that students be provided with a variety of resources and approaches to learning a new skill. In an undergraduate teacher preparation course in educational technology, we provided the PSTs with opportunities to (a) explore theory relevant to online instruction, (b) view examples, (c) practice teaching online in an authentic setting, (d) collaborate for broad understanding, and (e) reflect about online teaching.

The PSTs corresponded with Taiwanese partners who live on a geographically isolated island in A sia with an official language of $\mathrm{M}$ andarin $\mathrm{Chi}$ nese. Typically, Taiwanese learners of English-as-a-foreign-language (EFL) do not have much opportunity to communicate with native English speakers. In addition, one-on-one practice time and frequent or detailed discussion of cultural issues are limited. Foreign language learners need to practice their language skills in an authentic setting because learners acquire foreign languages through acts of communication rather than through isolated study of language forms and semantic structures. In order to meet the needs of the PSTs and of the Taiwanese English language learners, we formed our telecommunications partnership.

The use of telecommunications allowed for authentic acts of communication, one-on-one interaction, and detailed discussion. Those processes supported PSTs for learning how to teach online and how to reach diverse audiences and supported Taiwanese students for learning to express themselves in English and learn about A merican culture.

\section{The Correspondence Process}

The PSTs' assignment was to 1) experience and practice online teaching; 2) establish friendships with students of another culture; 3) exchange cultural information; 4) improve online communication skills; 5) identify the benefits and limitations of online learning; and 6) identify the benefits and limitations of online teaching. The A merican PSTs were assigned to correspond weekly and to send at least ten messages during two months. They began with an introduction of themselves and their intentions to provide instruction in English language or A merican culture. In a second message, they established the needs of their keypals by asking what their partners needed to learn and how they might help and by examining samples of their partners' writing to ascertain their capabilities. The rest of the correspondences (a minimum of eight messages) were supposed to include instruction in English language as well as A merican and Taiwanese cultures. PSTs began instructing their partners while attending to their capability levels.

The three collaborating teachers in Taiwan set the following objectives for their students: 1) write with A merican students about interesting topics; 2) improve English composition skills; 3) experience online learning; and 4) exchange cultural information. Similar to the PSTs' directions, Taiwanese students were to introduce themselves in a first letter. The second message was to explain their needs of assistance from their partners and to help the PSTs establish the learning goals that ranged from understanding Shakespeare's English, practicing conversational English, and reading novels, to becoming acquainted with A merican university life. The subsequent messages were to be interactions with the PSTs on the selected goals.

In addition, PST tutors and their Taiwanese EFL students could discuss online teaching, online learning, cultural issues, and technical problems on a W eb board set up as an open forum. These discussions were not part of the course's requirements and were not well attended.

\section{On nine Teaching}

To prepare the PSTs for the difficult task of online teaching, we facilitated an interactive presentation of related concepts, led face-to-face discussions about possible processes, provided supportive readings and example tutorial correspondences, and created a W eb site of resources. The digital pictures of the PSTs and the Taiwanese students on the Web site facilitated social presence during online correspondence. In addition, the Web site (http:// www. coe. tamu. edu/ /cifuent/classes/ edtc305/online. htm) included a downloadable presentation, the expected online correspondence process guidelines, a formative evaluation survey, sample correspondences, and samples of participants' reflections. PSTs read on topics such as effective facilitation of computer conferencing, interactivity in online environments, transactional distance, effective questioning, and text-based instruction ( see Figure 1). In addition, we used an electronic bulletin-board system to model online instruction in mock tutorials. PSTs "lurked" while we demonstrated delivery of online lesson cycles in language and social studies.

\section{On une Teaching Strategies}

$O$ nline teaching strategies used by the PSTs fell into the following categories:

- facilitative information,

- questions and answers from tutor to student,

- questions and answers from student to tutor,

- topic discussion,

- problem-solving, 
Figure 1. Homepage with support materials for preservice teachers

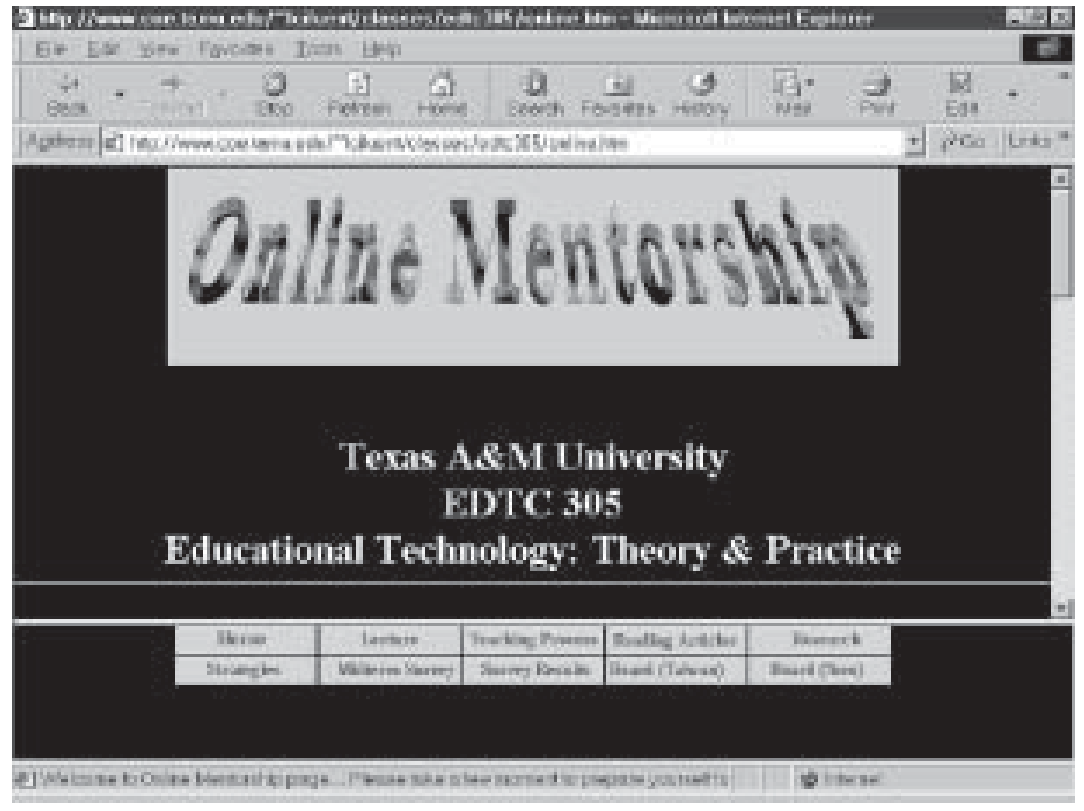

- critique of writing, and recommendations for metacognition.

The PST tutors provided facilitative information by including references to books or Web sites that they felt would be helpful for language learning. A Taiwanese student claimed that "Those Web sites... are interesting and I really can learn something from it." A s part of an explanation of $\mathrm{Hal}$ loween traditions, another PST provided a $\mathrm{H}$ alloween graphic.

Both PSTs and their Taiwanese students asked questions to learn from the correspondence. One question was meant to begin a dialog about journalistic approaches. A PST asked, "H ave you taken any journalism classes? If you have, do they teach you the same concepts [as described in an earlier message] or is journalism different there?" A nother question came up while a PST and her student touched upon the topic of politics, "W hat is the government like? Do you have anything like states?" Taiwanese students asked questions of their tutors to elicit information or skill learning. For example, "The first homework for our linguistics is gestures! Could you give some suggestions? Thank you first."

The bulk of the e-mail correspon- dences consisted of topic discussions. Topics included, but were not limited to, movie favorites, celebrations and holidays, travel experiences, school life, study habits, hobbies, religion, and personal philosophy. PSTs also engaged their students in problem-solving activities and many of these were quite creative and interesting as lessons. For instance, one PST gave his student verbal riddles to solve in writing. A very creative PST provided her student with directions to follow regarding scenarios that she also provided:

"In the following situations you are asked what you would say if you had to refuse someone. In the first one, it is someone who is a lower status person, the second is someone of equal status, and the third one is someone of higher status. For each of the situations, type in what you would say in order to turn down their request."

A nother PST gave a list of incorrect sentences for the student to correct and later provided feedback for further improvements of those sentences. Some PSTs critiqued their students' writing and helped them to correct mistakes in essays or e-mail messages. A $n$ easy approach to providing a critique involved highlighting language mistakes with numbers or asterisks and making corrections in replies to their students.

A few PSTs suggested metacognitive strategies for their students. For instance, a PST provided her student with this suggestion: " . . . for memorizing the dynasties, ... . put them in order on a timeline and write a couple of things you think of when you see the name." That same tutor also wrote advice to her student, "To help with sentences that I did not understand, sometimes, I would try to rewrite them in my own words."

\section{Onune Foreign Language LEARN ING}

The students in Taiwan were supplied with an orientation to the project along with rules and regulations regarding correspondence. Sample correspondence, results of previous connections, and suggestions for online learning and discussion topics were presented at their departmental Web site (http:// w w w. e n g. f ju, ed u. t w / cultural_connections. htm, see Figure 2).

\section{Onune Learning Strategies}

W hile communicating with the tutors and learning from them as well as from the online experience itself, the Taiwanese learners used a total of 10 learning strategies in the correspondence. Through the use of these strategies, the Taiwanese learners practiced writing understandable English to convey their thoughts and meanings to the U.S. tutors. The learning strategies were-

- responsive dialogue,

- translation,

- responding to tutors' questions,

- asking questions of tutors,

- explanation,

- elaboration,

- decision-making,

- self-reflection,

- metacognitive strategies, and

- transfer.

Several Taiwanese students re-em- 
phasized their tutors' main points by repeating and rephrasing these comments in their own messages as responsive dialogue. In one message, a Taiwanese student expressed her understanding of her tutor's feeling about the Bonfire tradition, which the tutor had thoroughly explained in her previous message.

A nother student summed up her tutor's opinions on dating and told her tutor that she held a different point of view:

I'm sorry to hear that you've broken up with your boyfriend which have been together for three years. Knowing that you only want to have fun and enjoy your life, I found something funny that the attitudes towards love between you and me are totally different.

W hen the Taiwanese students mentioned concepts or words in $\mathrm{M}$ andarin Chinese, they needed to translate them into English to assist the PSTs' comprehension. One Taiwanese student explained the meaning of her Chinese name, "My Chinese name is $\mathrm{H}$ sin-Yi, and it means the joy of heart. I guess my parents must want to wish me happiness in my life, so they gave it to me."

In order to express her gratitude to her tutor, a Taiwanese student trans-

Figure 2. Homepage with support materials for Taiwanese students

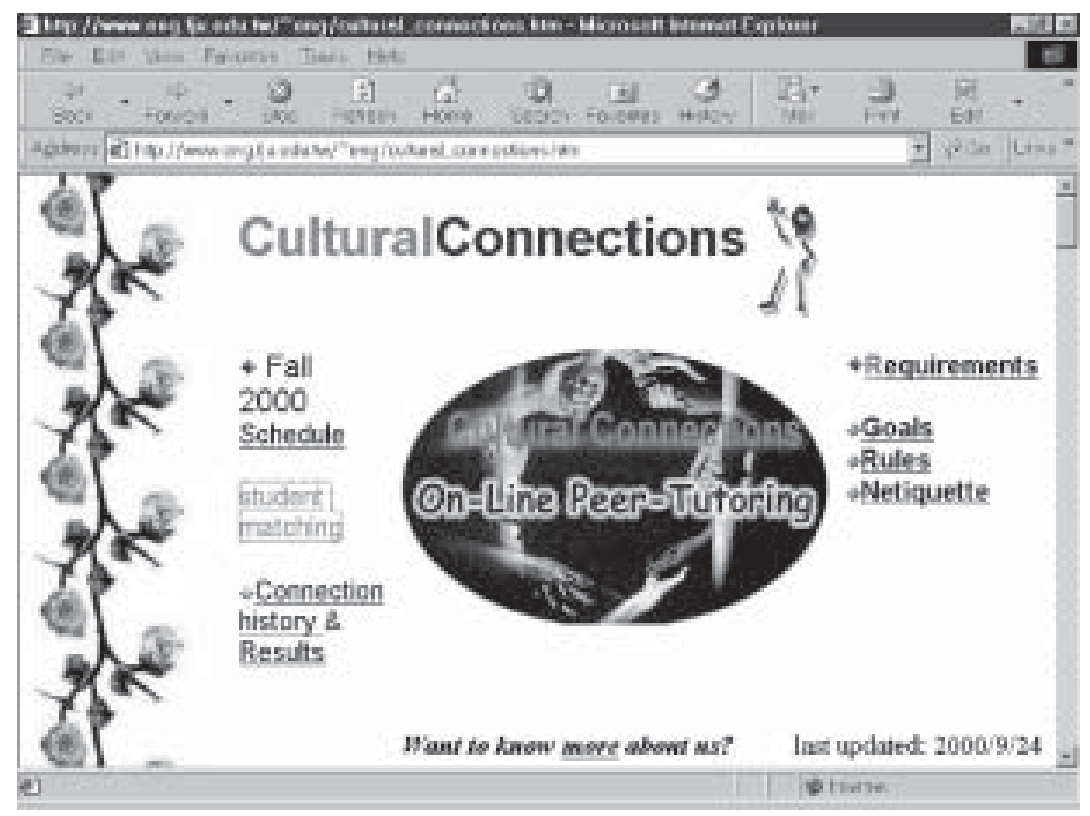

lated a Chinese proverb to show her appreciation. She typed in her message:

There is an old Chinese proverb. It is said that if you could take the same boat with somebody, it's because that you had cultivated your moral character for ten years in your previous life. If you could be someone's wife/husband, it's because you had cultivated your moral character for one hundred years in your previous life. This proverb tells us to get along well with people you know, for it's precious to have this relationship. What I want to tell you is that I am thankful to be your pen pal.

Students were able to tidy up their thoughts or initiate reflections by responding to tutors' questions. The PSTs' questions functioned as advance organizers or discussion reviews for the learners. O ne PST explained the tradition of Thanksgiving to her student and then asked him two questions related to this holiday:

Now, here are some questions you can answer:

1. If you were celebrating Thanksgiving this year in A merica, what would you list as the things that you have to be thankful for?
2. Is there a holiday celebrated in Taiwan that is similar to Thanksgiving or deals with the abundance of a harvest? If so, tell me about it.

The Taiwanese student ignored his tutor's first question and responded to the second question:

We would have a day off here and we would go back to where our ancestors live, usually in the country. W e're taught that it's the day that we come back to where we came from and be grateful for our grandfather or great grandfather's efforts since they'd been worked so hard to establish a home from which we all come from.

W hile PSTs' questions evoked the students' reflective thinking and explanation of their views, students also used this questioning strategy to draw new information from their tutors. For example, when a pair were discussing Shakespeare, the Taiwanese student wanted to know more about this great writer. She asked, "W hat do you think Shakespeare as a person? A nd why he has so much influence to British literature?" A nother student asked her tutor to clarify the differences between two words, "Few weeks ago, our class discussed about "love or infatuation.' How do you define these words?" A nd yet another asked her tutor to clarify the meaning of hip-hop.

While explaining their views or thoughts, the Taiwanese students learned to write in organized paragraphs and to use appropriate words in English to convey their meanings to the tutors in adequate forms. For instance, a PST mentioned that she would like to get to know her Taiwanese student and her country better, so the student explained how people are named in Taiwan: "These two words [of my name] both mean the fragrance of flowers... I In Chinese tradition, lots of names are named by fortunetellers."

The Taiwanese students also elaborated on what they were learning. They related new information to other concepts or to their previous experiences or knowledge either directly or by cre- 
ating analogies. O ne student and her tutor were comparing the Taiwanese culture to the U.S. culture. This student was trying to analyze the personalities of A sian people by relating them to her previous knowledge:

I feel that the reason why A sians may be seen as inferior individuals is because they were one of the last cultures to start a movement for equality that was inspired by other ethnic cultures. Because of this somewhat late movement, our culture has not been able to adapt to this change, like our culture has not adapted to women being paid the same as men. For example, if you have ever seen a movie or a television who that had an A sian character, nine times out of ten the A sian character is depicted as being mathematicians, naive, and speaks with broken English.

\section{Participants emphasized the importance of telecommunications as a means of having experiences teachers and learners cannot have otherwise.}

Learners practiced communication and negotiation skills using a decisionmaking strategy. In the connection, the tutors were required to assist their students in defining learning goals and objectives for the connection. Many pairs took several messages to reach a final decision. M ost pairs showed their respect for each other by consulting with each other before moving forward with instruction. For instance, one student made a request to her tutor: "I would like to talk about the music, can you tell me what kind of music you like? I would like to hear that." The tutor agreed to speak on this topic and they had a long conversation about country music and Christmas music.

$M$ any students reflected on their own learning of EFL or reading of literature. For instance, one student reflected on her improper use of the English language:
My English was sometimes like Chinese English. I translated Chinese to English directly, so sometimes I could not have myself understood by native English speakers. Therefore, I think it is still a long way to go when I can master English.

In addition, many partners reflected on the meaning of life as a consequence of the Taiwanese earthquake and the Texas $A \& M$ bonfire tragedy experienced by each group during one semester of the partnership.

The Taiwanese students demonstrated conscious access to their own metacognitive strategies. For instance, they illustrated in their messages what was or could be done to help them achieve certain goals. This indicated that they were conscious of their higher-level thinking processes. For example, one student wrote:

\section{Benefits of O nune Education in Traditional Classes}

Most participants' reactions to their first online teaching and learning experiences were positive. The participants identified the following benefits

- online experience,

- time for details and individualized instruction,

- authentic foreign language learning, and

- cultural exchange.

Participants emphasized the importance of telecommunications as a means of having experiences teachers and learners cannot have otherwise. The process helped PSTs to acquire skills of effective online teaching as well as teaching in general. In addition to experiencing online teaching, the correspondence allowed PSTs to be responsive to an individual learner's needs and interests and provided experience with personalized instruction. The Tai wanese students valued the opportunity for authentic English language learning through writing practice with an A merican reader who could provide writing models and give feedback. Students suggested that the writing skills they learned through the correspondence transferred to increased speaking skill. In addition, all participants benefited from the opportunity for cultural exchange that helped the PSTs learn how to teach students of A sian descent and helped the Taiwanese increase their knowledge of A merican culture. from the connection to a context beyond the connection. For example, one student greeted her tutor with the word "Howdy," which she picked up from her peer who was connected to another tutor. In a different pair, the Taiwanese student expressed happiness that the tutor in her connection was an expert in A merican football and taught her everything about it. Later, she was able to compare A merican football to English football in one of her compositions.

\section{Limitations of O nune Education}

Participants identified five limitations associated with the online teaching and learning experience:

- vulnerability to technical failure,

- constraints of e-mail and W eb boards,

- dependence on an unresponsive partner,

- sense of detachment, and

- time.

First, although technical failure oc- 
curred in only one set of key-pals, it still provided a minor psychological nuisance, as others became aware of the possibility of electronic difficulties. Second, e-mail and W eb boards as correspondence tools restricted both the PSTs and the Taiwanese students to transfer text-based messages. O nly one PST sent a graphic attachment, the festive $\mathrm{H}$ alloween card. PSTs expressed frustration regarding technical difficulty of using audio or visual aids to help their students. The Taiwanese students also felt limited by electronic correspondence. " $M$ any things cannot be shown through writing."

Third, slow or no responses from partners on both ends led to frustration. A partner on one end might have been satisfied while a partner on the other end wasn't. Both the PSTs and their Taiwanese students complained of lack of responsiveness on the part of their partners. To avoid lack of responses from participants, Warschauer (1995) suggested connecting students to multiple partners either in the same class or several classes. A fourth limitation, sense of detachment, created difficulties for the PSTs as they tried to assess student needs and progress. A PST said "I found it difficult to assess exactly what my student needed to learn." "I don't know if the student is really learning... when you are teaching students over e-mail, you cannot see if the 'light' of understanding is going off as you can when you are teaching faceto-face." "We are limited because we don't know what is happening on the other side." Similarly, the Taiwanese students lacked clarity regarding their PSTs' expectations. "I find it is difficult to ask questions because I don't know what does she know."

The last limitation, time, affected the correspondence in two very different ways. First, time zone differences often associated with international collaboration interfered with participants' abilities to meet in real-time, and second, online teaching and learning proved to be time consuming for all participants.

The time zone difference restricted classes from meeting synchronously through videoconferencing or electronic chats during class time. PSTs expressed the wish to see their online students and communicate in realtime. A lthough they could have met as partners via desktop videoconferencing outside of class time and the PSTs had received instruction in that process, none of the partners took advantage of that capability.

PSTs and Taiwanese learners needed a great deal of time to accomplish their tasks while the instructors spent an inordinate amount of time grading the activity. Both PSTs and Taiwanese students expressed the wish that they could correspond for a longer period of time. Due to the differences in the length and timing of a semester in both countries, the connection could be established for a maximum of two months within the context of the courses. H owever, parties at both sites could, of course, choose to continue their online teaching and learning experiences independent of the courses if

O nce the learners tore down the communication barriers, they were able to work successfully with each other practicing active learning, critical inquiry, and reflective thinking.

they wished. Indeed, several PSTs mentioned that they and their partner planned to continue correspondence beyond the semester.

\section{Conclusions}

W hy build telecommunications partnerships into traditional courses? In the case described here, the telecommunications partners gained knowledge, skills, and awareness through their online connection and met the courses' objectives through distance correspondence. PSTs learned about teaching online as well as teaching in general by applying instructional strategies with real learners. Likewise, the Taiwanese students received instruction from native English language speakers and were able to explore the A merican culture. Such authenticity could only be attained through application of distance technologies. A s a result of their experiences, participants constructed elaborate understandings of the cultures of teaching and foreign language learning.

H owever, the ben efits went beyond the main purposes of the project. Several partners established lasting relationships that have continued long after the course objectives were met and continued to support learning long after the students completed the courses. Friendships were formed among people of two dramatically different cultures. During the asynchronous communication between the U nited States and Taiwan, the learners eliminated their shyness and started to express their thoughts freely.

Once the learners tore down the communication barriers, they were able to work successfully with each other practicing active learning, critical in-

quiry, and reflective thinking.

We have identified categories of online teaching strategies and learning strategies that can guide further teacher preparation and foreign language learning efforts. Inexperienced PSTs used topic discussion and leading questions as their dominant online 
Continued from page 13

teaching strategies. Inexperienced Taiwanese learners tended to use responding to tutors' questions, asking questions of tutors, and explanation more than other strategies. We recommend that online teachers and their students become acquainted with our identified online teaching and learning strategies in preparation for effective learning in the virtual environment. U se of the full range of strategies should prove most effective in online environments. No doubt, other strategies will emerge with more experience.

Based on our experiences we make these recommendations for connections:

- instructors should be available to facilitate and moderate interactions when necessary;

- technical support should be provided for inclusion of audio, graphics, and video exchange as well as videoconferencing;

- correspondence should extend for as long as the course schedule allows;

- a list of frequently asked questions (FA Q) and models of exchanges should be provided; and

- guidelines for how to teach, learn, and communicate interculturally should be provided.

Educational endeavors are wise to infuse authentic activities facilitated by telecommunications technologies into their curricula. Such infusion becomes possible as more schools go online. Due to the positive reactions to the online teaching and learning experience in the partnership described here, we will continue collaborating with Taiwanese professors in pairing students for online teaching and learning. In addition, we hope to expand our connections to create not only a network of international partners but also multicultural partners within the U nited States.

\section{References}

Q uality Education Data. (1999, July). School with online access: School survey. Internet usage in public schools, $4^{\text {th }}$ edition. [O n-line]. Retrieved from E-mail: QED_OnLine@geddata.com.

Romiszowski, A. J., \& Ravitz, J. (1997). Computer-mediated communication. In C.R. Dills and A.J. Romiszowski (Eds.), Instructional development paradigms (pp.745-768). Englewood Cliffs, NJ: Educational Technology Publications.

W arschauer, M . (1995). E-mail for English teaching. A lexandria, VA: Teachers of English to Speakers of 0 ther Languages.

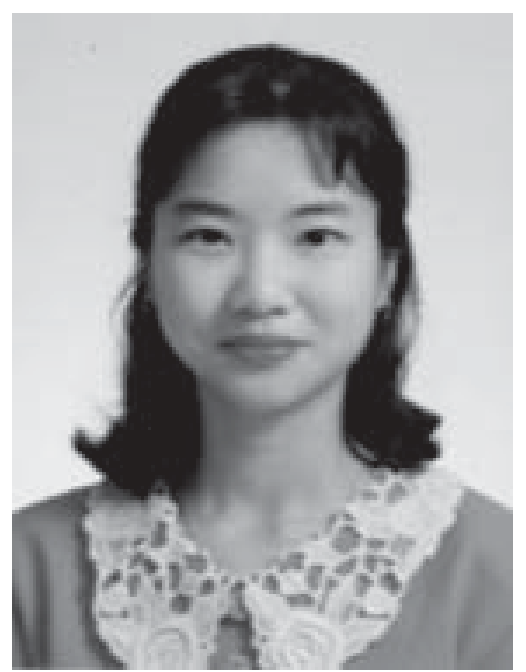

Yu-Chih Doris Shih recently earned her PhD from the Educational Technology Program at Texas A\& M University. Her research interests are in foreign language and cultural learming with technologies.

Lauren Cifuentes is an assistant professor in the Educational Technology Program at Texas A\& M University where she teaches instructional design and computer graphics. Her primary research interest is in the design of telecommunications partnerships.

\section{Online membership renewal is now available! Just a few clicks of your mouse and you can renew your membership in A ECT. It's fast, easy, and secure. $\mathrm{G} 0$ to www.aect.org.}

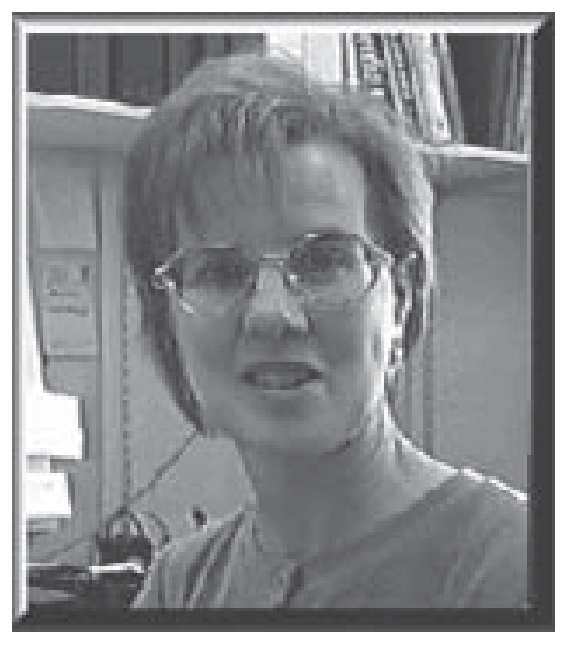

